This item was submitted to Loughborough's Research Repository by the author.

Items in Figshare are protected by copyright, with all rights reserved, unless otherwise indicated.

\title{
Damping of flexural vibrations in plates containing ensembles of tapered indentations of power-law profile
}

PLEASE CITE THE PUBLISHED VERSION

http://dx.doi.org/10.1121/1.4776153

\section{PUBLISHER}

(C) Acoustical Society of America (Published by the Acoustical Society of America through the American Institute of Physics)

\section{VERSION}

VoR (Version of Record)

\section{LICENCE}

CC BY-NC-ND 4.0

\section{REPOSITORY RECORD}

Bowyer, E.P., D.J. O'Boy, and Victor V. Krylov. 2013. "Damping of Flexural Vibrations in Plates Containing Ensembles of Tapered Indentations of Power-law Profile". figshare. https://hdl.handle.net/2134/11512. 
This item was submitted to Loughborough's Institutional Repository (https://dspace.lboro.ac.uk/) by the author and is made available under the following Creative Commons Licence conditions.

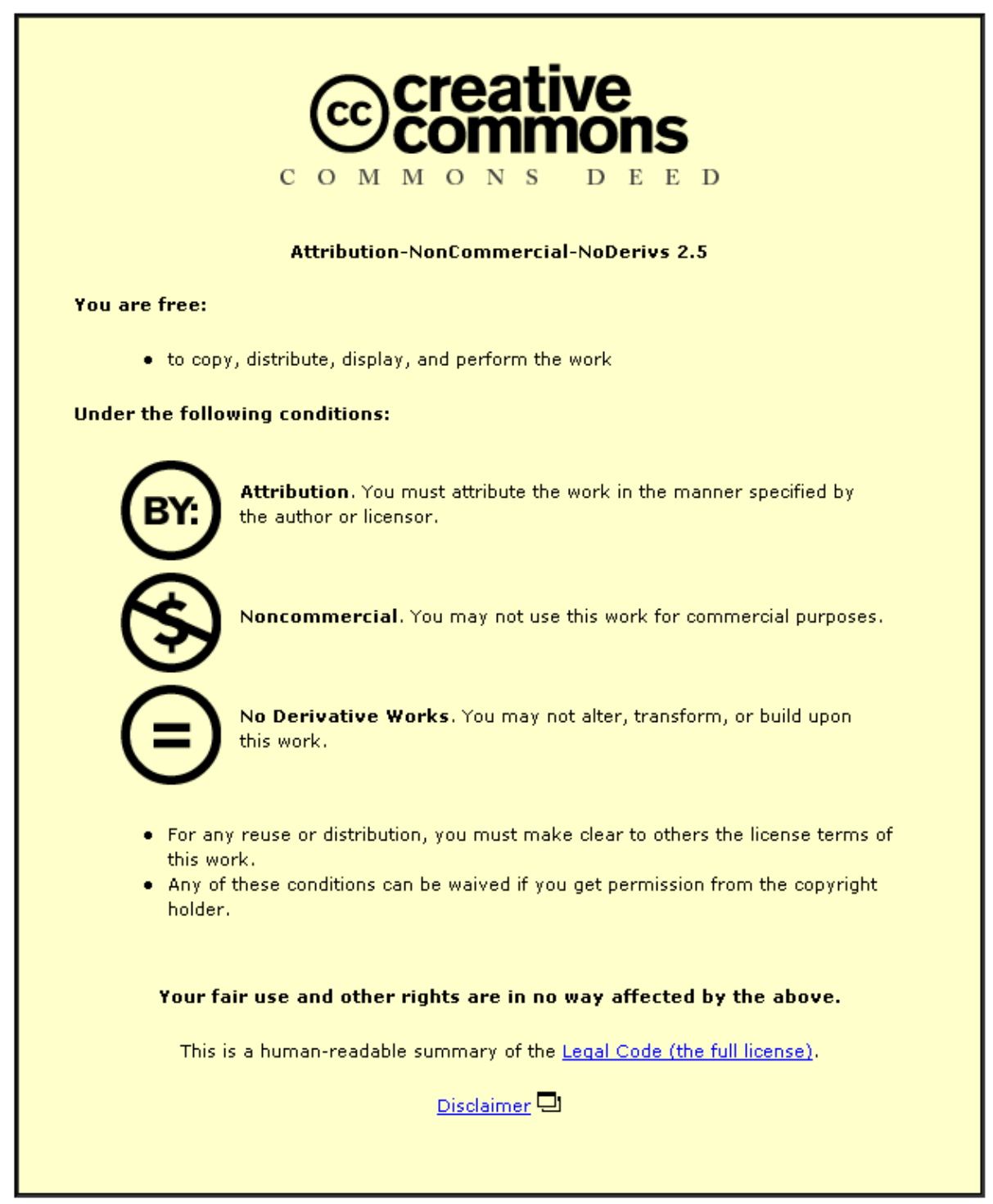

For the full text of this licence, please go to: http://creativecommons.org/licenses/by-nc-nd/2.5/ 


\section{Proceedings of Meetings on Acoustics}

164th Meeting of the Acoustical Society of America

Kansas City, Missouri

22 - 26 October 2012

Session 4aEA: Engineering Acoustics

\section{4aEA9. Damping of flexural vibrations in plates containing ensembles of tapered indentations of power-law profile}

Elizabeth P. Bowyer, Daniel J. O’Boy and Victor V. Krylov*

*Corresponding author's address: Aeronautical and Automotive Engineering, Loughborough University, Ashby Road, Loughborough, LE11 3TU, Leicestershire, United Kingdom, V.V.Krylov@lboro.ac.uk

In this work, we report experimental results on damping flexural vibrations in rectangular plates containing tapered indentations (pits) of power-law profile, the centres of which are covered by a small amount of absorbing material. In the case of quadratic or higher-order profiles, such indentations materialise two-dimensional acoustic 'black holes' for flexural waves. Initially, the effects of single pits have been investigated. It has been found that, in order to increase the damping efficiency of power-law profiled indentations, their absorption crossections should be enlarged by drilling a central hole of sufficiently large size $(14 \mathrm{~mm})$, while keeping the edges sharp. Such pits, being in fact curved power-law wedges, result in substantially increased damping. The next and the major part of this investigation involved using multiple indentations in the same rectangular plates to increase damping. Plates with combinations from two to six equal indentations have been investigated. The results show that, when multiple indentations are used, the associated damping increases substantially with the increase of a number of indentations. For the plate with 6 indentations, the resulting damping becomes comparable if not greater than that achieved by a wedge of power-law profile.

Published by the Acoustical Society of America through the American Institute of Physics 


\section{Introduction}

Damping of structural vibrations remains a challenging problem for different branches of engineering, especially for aeronautical and automotive applications. Passive damping of structural vibrations is usually achieved by adding layers of highly absorbing materials to the structure in order to increase energy dissipation of propagating (mostly flexural) waves ${ }^{1-3}$. An alternative approach, that can be used to suppress resonant vibrations of different structures, is based on reduction of reflections of structural waves from their edges ${ }^{4}$.

To implement the approach described in Ref 4 in a more efficient way, a new method of damping flexural vibrations based on the so-called 'acoustic black hole effect' has been recently developed and investigated ${ }^{5-7}$. This method has been initially applied to one-dimensional plates of power-law profile (wedges), the wedge tips having to be covered by narrow strips of absorbing layer. Ideally, if the power-law exponent is equal or larger than two, the flexural wave never reaches the sharp edge and therefore never reflects back ${ }^{5-8}$, which implements an 'acoustic black hole'. However, ideally sharp wedges do not exist in reality, therefore the presence of even small strips of damping layers at free edges is paramount to obtain very low reflections from the tips, which constitutes the acoustic black hole effect ${ }^{5-7}$. It has been established theoretically ${ }^{5,6}$ and confirmed experimentally ${ }^{7}$ that this method of damping structural vibrations is very efficient even in the presence of edge truncations and other imperfections.

The natural development of research into methods of damping structural vibrations using the acoustic black hole effect was investigation of twodimensional tapered indentations (pits) of power-law profile, with the addition of a small amount of absorbing material ${ }^{9}$. In the case of quadratic or higher-order profiles, such pits materialise two-dimensional acoustic black holes for flexural waves. To understand basic principles of operation of such 'black holes', a geometrical acoustics approach to analysing flexural wave interaction with powerlaw indentations has been developed ${ }^{9}$. This approach shows that, if a flexural wave propagates towards a profiled indentation, some of the incident rays become captured and their reflection from the free edge of a small internal hole in the pit can be calculated in the same way as in the case of one-dimensional wedges.

First experimental investigations of two-dimensional acoustic black holes have been described in Refs 10-12 dealing with damping of flexural vibration in elliptical plates. It has been demonstrated in these papers that such indentations can be very efficient dampers if they are placed in one of the plate's foci. Vibrations of circular plates with indentations of power-law profile placed in the centres have been investigated in Ref 13 both theoretically and experimentally. In Ref 14, vibration damping in rectangular plates with circular indentations of power-law profile has been considered experimentally. It has been shown in these two papers 
that such indentations also act as efficient vibration dampers. It should be noted that using circular indentations inside plates offers a range of advantages in comparison with wedges of power-law profile attached to plate edges. First of all, the potentially dangerous sharp edges of power-law wedges are eliminated, so that all exposed sides of the plate are of the same nominal thickness, which brings a safety benefit. Secondly, two-dimensional pits can be applied to suppress just some selected resonant peaks, when placed in certain positions.

The focus of the present work is on the experimental investigation of damping flexural vibrations in rectangular plates containing ensembles (arrays) of twodimensional tapered indentations (pits) of power-law profile. It is expected that such ensembles will amplify the action of a single indentation, which will increase the efficiency of damping.

\section{Experimental samples and procedure}

Experimental samples in the present work were manufactured from $5 \mathrm{~mm}$ thick hot drawn mild steel. Dimensions of the produced rectangular plates were $400 \times 300$ $\mathrm{mm}$. All indentations had profiles with the power-law exponential $\mathrm{m}=4$.

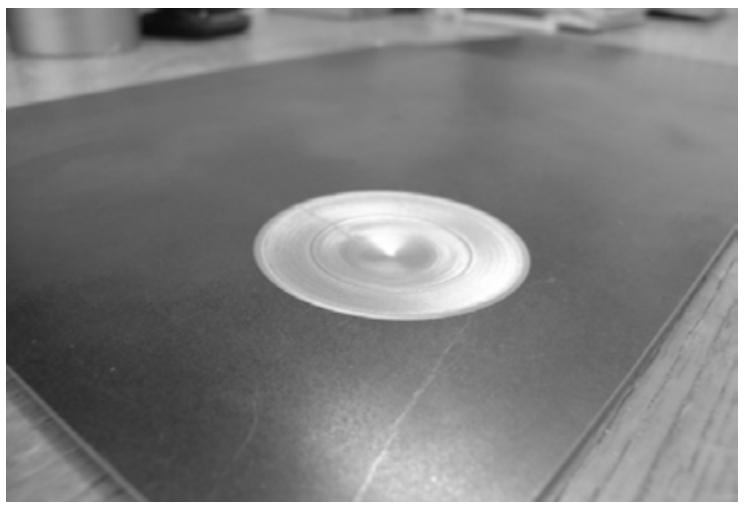

Figure 1: Plate containing a circular indentation with no central hole.

A CNC (Computer Numerically Controlled) milling machine operating at a cutter speed of $1200 \mathrm{rpm}$ was used to produce circular fourth-power indentations into the plates. Three types of experimental samples were produced for this investigation, a plate with a singular circular indentation (Figure. 1), a plate with a singular circular indentation with a drilled central hole, and plates containing multiple profiled circular indentations with central holes, two indentations, Figure 2(a), four indentations, Figure 2(b) and, six indentations, Figure 2(c). 
There are three main problems encountered when utilizing this method of manufacturing. The first being that at the centre of the indentation, where the material thickness is less than $0.4 \mathrm{~mm}$ and the machining stress and resulting heat are high, there may be blistering leading to inaccurate results during experimental testing. Secondly, it is the formation of a machine line, as the cutters movement through the indentation is computer controlled. It merely moves from one programmed height to another, gouging the material and creating a raised line in the indentation. Finally, additional damage can occur when a hole is drilled into the centre of the circular indentation. Due to the small thickness of the material at this point, it is more susceptible to tearing.

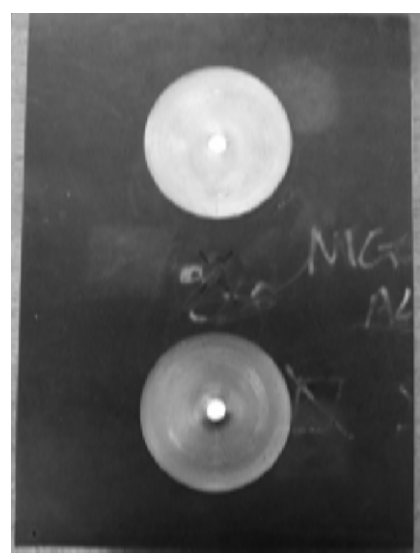

(a)

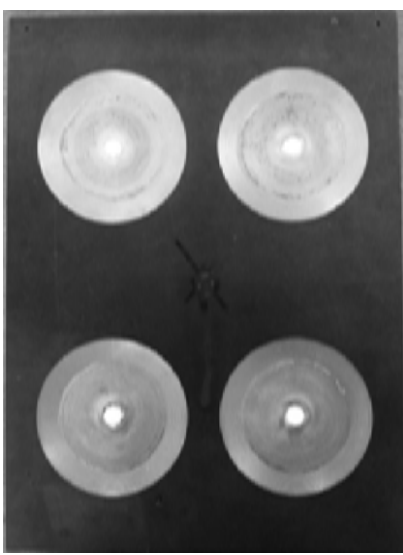

(b)

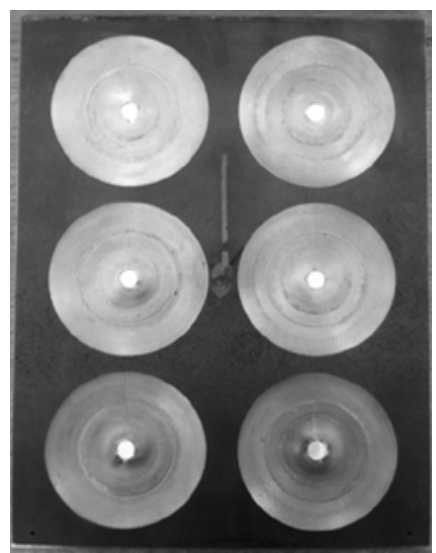

(c)

Figure 2: Profiled circular indentations with central holes: (a) - two indentations; (b) - four indentations; (c) - six indentations.

The experimental set-up has been designed to allow nearly free vibration of the sample plates (i.e. to eliminate clamping of edges), take the weight off the plate edges and introduce minimal damping to the system, Figure. 3a. The excitation force was applied to the centre of the plate via an electromagnetic shaker attached to the plate using 'glue' and fed via a broadband signal amplifier. The response was recorded by an accelerometer (B\&K Type 4371) that was attached to the upper surface, directly above the force transducer (B\&K Type 8200), also via 'glue' (Figure 3b).

The acquisition of the point accelerance was utilised using a Bruel \& Kjaer 2035 analyser and amplifier over a frequency range of $0-9 \mathrm{kHz}$. The results presented in this paper have been averaged over five measurements in order to 
ensure a statistically accurate representation of the point accelerance. A plain reference plate was used for comparison.
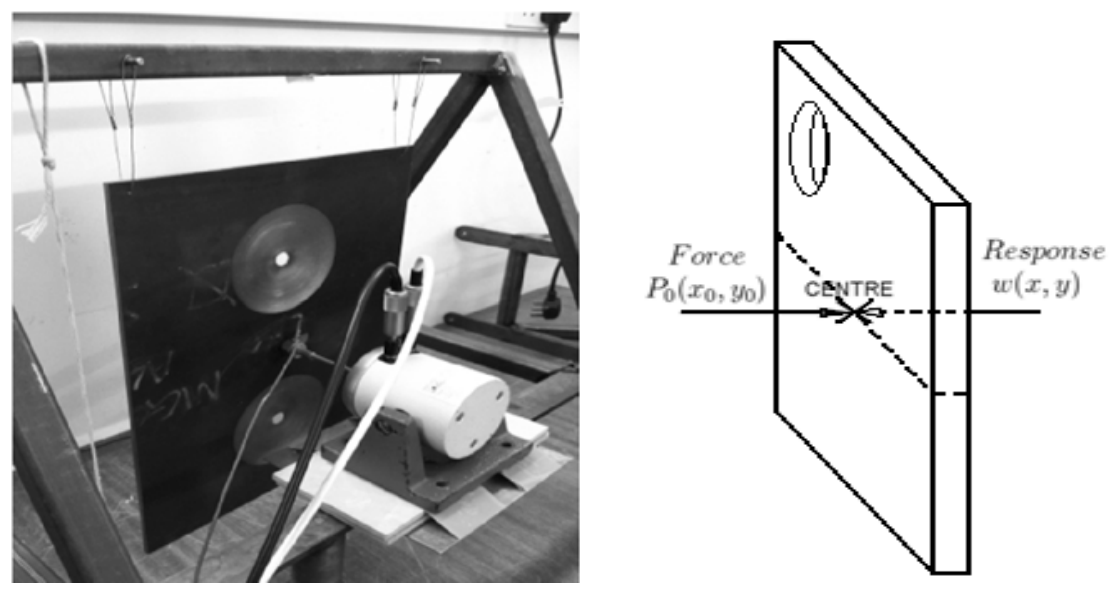

Figure 3: (a) Experimental setup, (b) Locations of the shaker (Force) and of the accelerometer (Response) on an experimental sample.

\section{Results and discussion}

\subsection{A single circular indentation with and without a central hole}

Initial measurements with a single circular indentation without a central hole (i.e. with a just protruding indentation) demonstrated that in this case there were no noticeable damping effects when compared to a reference plate. From these initial observations it has been decided that a central hole has to be drilled into the centre of the indentation. Note that, due to manufacturing limitations at the centre of the circular pit, there was an area of almost equal thickness of about $0.4 \mathrm{~mm}$ that extends from the central point out to a diameter of approximately $3 \mathrm{~mm}$. Thus, a hole of $2 \mathrm{~mm}$ diameter could therefore be drilled without affecting the minimum tip thickness. Measurements of frequency responses for such indentations have been carried out in Ref 14 for profiles with $m=2$. The results showed that the effect of drilling a hole in the centre of the circular indentations increases the 
overall losses at higher frequencies, most likely due to increased absorption crosssection of such indentations.

In attempts to improve the damping efficiency of the profiled indentations, the central hole size was increased progressively by $2 \mathrm{~mm}$ until a central hole size of $14 \mathrm{~mm}$ and an indentation diameter of $100 \mathrm{~mm}$ was achieved. The results have demonstrated an increased efficiency of damping for indentations with larger central holes ${ }^{14}$. In the present work, similar investigations have been carried out for indentations of power-law profiles with $\mathrm{m}=4$.

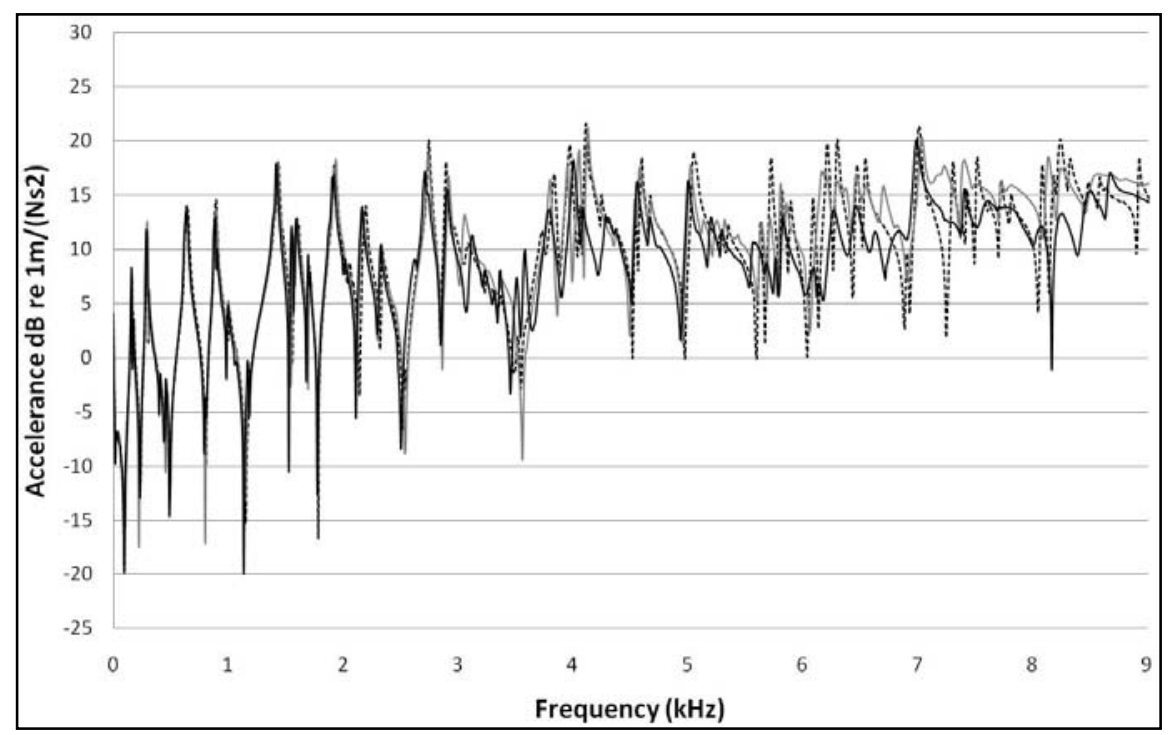

Figure 4: Measured accelerance for a damped circular indentation with a $14 \mathrm{~mm}$ central hole (solid black line) compared to a damped circular indentation with a $2 \mathrm{~mm}$ central hole (dashed line) and a $10 \mathrm{~mm}$ central hole (solid grey line).

Figure 4 shows a direct comparison between the circular indentations of power-law profile with $\mathrm{m}=4$ having $2 \mathrm{~mm}, 10 \mathrm{~mm}$ and $14 \mathrm{~mm}$ central holes. Below $3.5 \mathrm{kHz}$, the responses of all three samples are almost identical. In all other regions the 14 $\mathrm{mm}$ central hole has an increased damping performance compared to the $2 \mathrm{~mm}$ and $10 \mathrm{~mm}$ central hole. Increasing the central hole diameter increases damping performance of the circular indentation as it increasingly resembles a curved wedge as the diameter of the central hole is enlarged and the curvature of the free edge diminishes (see also Ref 14 for indentations of power-law profiles with $\mathrm{m}=2$ ). The addition of a damping layer increases the damping performance of the indentation up until $8 \mathrm{kHz}$, after which it has a reduced effect. This pattern of varying damping 
is consistent with the theory of power-law profiled wedge damping ${ }^{5-7}$. The greatest increase in damping performance was achieved in the frequency range between $3.5-8 \mathrm{kHz}$, when a damping layer was attached.

A comparison of the results for a profiled circular indentation $(\mathrm{m}=4)$ with a 14 $\mathrm{mm}$ central hole with an additional damping layer to the results for a plain reference plate are shown in Figure 5. Again, Figure. 5 shows that below $2 \mathrm{kHz}$ the circular pit provides little to no damping. In the region of $3.8-9 \mathrm{kHz}$, damping varies between $1-10 \mathrm{~dB}$, with the maximum damping occurring at $6.5 \mathrm{kHz}$.

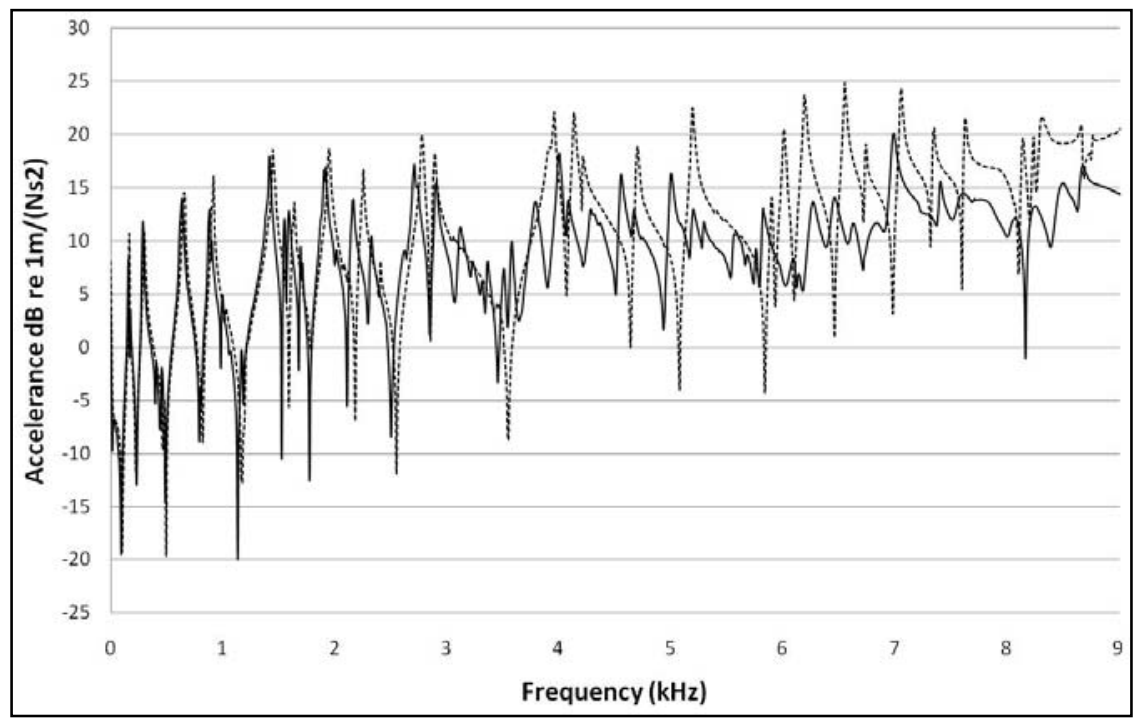

Figure 5: Measured accelerance for a profiled circular indentation with a $14 \mathrm{~mm}$ central hole with an additional damping layer (solid line) compared to a reference plate (dashed line).

\subsection{Ensembles of circular indentations of power-law profile}

In this section, the results of increasing the number of damped circular indentations on a centrally excited plate for two, four and six profiles are presented, when compared to each other and a reference plate. The diameters of the circular indentations were $114 \mathrm{~mm}$ and the central holes were $14 \mathrm{~mm}$ in diameter.

Figure 6 shows the results for the plates containing two, four and six profiled circular indentations with $14 \mathrm{~mm}$ central holes and additional damping layers. It is 
seen that the greater the number of indentations in the plate, the greater the damping obtained. The frequency at which a substantial reduction in peak amplitude is first seen decreases as the number of indentations increases. For the two, four and six indentation plates this occurs at approximately 4,3 and $2 \mathrm{kHz}$ respectively, as the lower frequency mode shapes start to correspond more closely with parts of the indentations patterns. After $4 \mathrm{kHz}$, it can be seen that the response clearly flattens increasingly with indentation number, thus increasing the level of broad band damping achieved compared to the high level of damping perceived at a specific frequency seen in the one indentation plate.

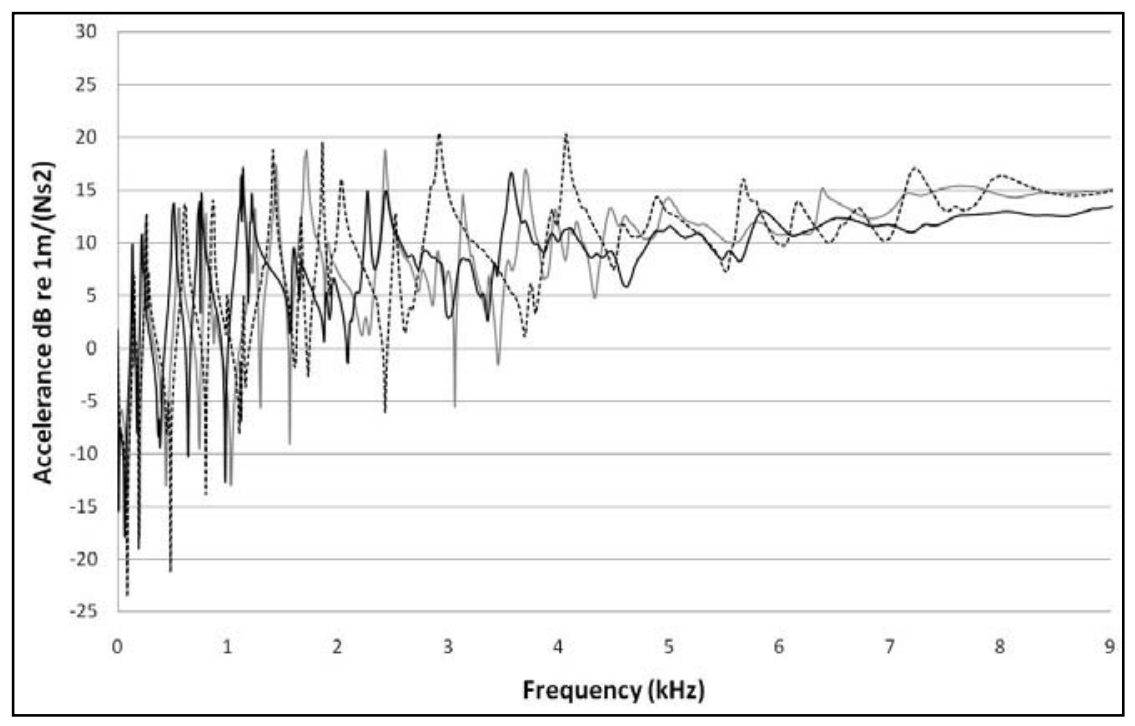

Figure 6: Measured accelerance for a plate containing two (dashed line), four (solid grey line) and six (solid black line) profiled circular indentations with $14 \mathrm{~mm}$ central holes and added damping layers.

The results for a plate containing six profiled circular indentations with $14 \mathrm{~mm}$ central holes and additional damping layers, compared to a reference plate, are shown in Figure 7. This configuration by far has the greatest damping at higher frequencies out of the samples tested, and it even outperforms a wedge of the same power-law profile. Below $1 \mathrm{kHz}$ there is little to no damping, as expected, and between $1-2 \mathrm{kHz}$ a reduction of peak amplitude by up to $4 \mathrm{~dB}$ is observed, after which damping increases until almost all peak responses are flattened. A maximum damping of $14 \mathrm{~dB}$ occurs at $6.5 \mathrm{kHz}$, this shift in the frequency at which maximum 
damping occurs is due to the reduction in mass and stiffness of the plates as the number of indentations increases.

As expected, the damping performance of the plates containing a singular indentation is not greater than the performance of $1 \mathrm{D}$ wedges of the same profile. However, the multiple indentation plates were able to compete with the damping performance provided by the tapered wedges. The position of the holes is linked to performance, and different combinations may result in greater levels of damping.

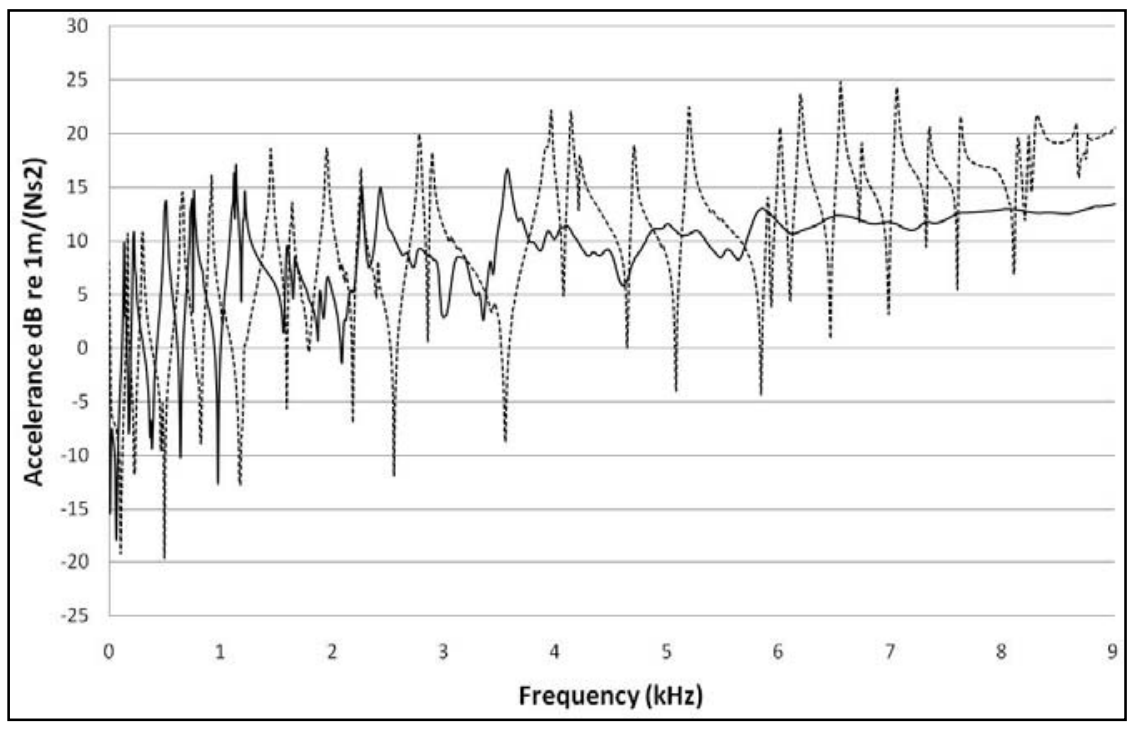

Figure 7: Measured accelerance for a plate containing six profiled circular indentations with $14 \mathrm{~mm}$ central holes and additional damping layers (solid line), as compared to a reference plate (dashed line).

\section{Conclusions}

It has been demonstrated in this paper that, in order to increase damping performance of a single indentation of power-law profile, its absorption crosssection has to be enlarged by increasing the size of the central hole in the indentation up to $14 \mathrm{~mm}$, while keeping the edges sharp. As expected, such indentations, being in fact curved power-law wedges, resulted in substantially increased damping performance that was comparable with that achieved by onedimensional wedges of power-law profile. 
As expected, the introduction of ensembles (arrays) of circular indentations of power-law profile into rectangular plates clearly increases the damping performance of such indentations. As the number of indentations increases so does the damping performance. The six indentation sample demonstrated an increase in resonant peak damping over a large frequency range. The obtained results show that using ensembles of circular indentations of power-law profile with large central holes can be very efficient method of damping flexural vibrations in platelike structures.

\section{Acknowledgements}

The research reported here has been partly supported by EPSRC grant EP/F009232/1.

\section{References}

${ }^{1}$ D.J. Mead, Passive vibration control, Wiley, Chichester (1998).

2 M. Heckl, L. Cremer, E. Ungar, Structure borne sound, 2nd Edition, SpringerVerlag, Berlin (1988).

${ }^{3}$ D. Ross, E. Kerwin, E. Ungar, Damping of plate flexural vibrations by means of viscoelastic laminae, In: Structural Damping (Ruzicka, J. E., ed) Vol. 3 (1959) 44-87.

${ }^{4}$ C. Vemula, A.N. Norris, G.D. Cody, Attenuation of waves in plates and bars using a graded impedance interface at edges, Journal of Sound and Vibration, 196 (1996) 107-127.

5 V.V. Krylov, F.J.B.S. Tilman, Acoustic black holes for flexural waves as effective vibration dampers, Journal of Sound and Vibration, 274 (2004) 605619.

${ }^{6}$ V.V. Krylov, New type of vibration dampers utilising the effect of acoustic 'black holes', Acta Acustica united with Acustica, 90 (2004) 830-837.

${ }^{7}$ V.V. Krylov, R.E.T.B. Winward, Experimental investigation of the acoustic black hole effect for flexural waves in tapered plates, Journal of Sound and Vibration, 300 (2007) 43-49.

${ }^{8}$ M.A. Mironov, Propagation of a flexural wave in a plate whose thickness decreases smoothly to zero in a finite interval. Soviet Physics - Acoustics, 34, 318-319, 1988.

${ }^{9}$ V.V. Krylov, Propagation of plate bending waves in the vicinity of one- and twodimensional acoustic 'black holes', in: Proceedings of the ECCOMAS International Conference on Computational Methods in Structural Dynamics and 
Earthquake Engineering (COMPDYN 2007), Rethymno, Crete, Greece, 13-16 June 2007, [CD-ROM].

${ }^{10}$ F. Gautier, J. Cuenca, V. V. Krylov, L. Simon, Experimental investigation of the acoustic black hole effect for vibration damping in elliptical plates (Abstract for the Conference "Acoustics 08", Paris, France, June 2008), Journal of the Acoustical Society of America, 123 (5) (2008) 3318.

${ }^{11}$ V. Georgiev, J. Cuenca, M. A. Molerón-Bermúdez, F. Gautier, L. Simon, V. V. Krylov, Numerical and experimental investigation of the acoustic black hole effect for vibration damping in beams and elliptical plates, In: Proceedings of the European Conference on Noise Control “Euronoise 2009”, Edinburgh, UK, 26-28 October 2009, [CD-ROM].

${ }^{12}$ V. B. Georgiev, J. Cuenca, F. Gautier, L. Simon, V. V. Krylov, Damping of structural vibrations in beams and elliptical plates using the acoustic black hole effect, Journal of Sound and Vibration, 330 (2011) 2497-2508.

${ }^{13}$ D. J. O'Boy, E. P. Bowyer, V. V. Krylov, Damping of flexural vibrations in thin plates using one and two dimensional acoustic black hole effect, In: Proceedings of the $10^{\text {th }}$ International Conference on Recent Advances in Structural Dynamics (RASD 2010), Southampton, UK, 12-14 July 2010, [CD-ROM].

${ }^{14}$ E.P. Bowyer, D.J. O'Boy, V.V. Krylov, F. Gautier, Experimental investigation of damping flexural vibrations using two-dimensional acoustic 'black holes', in: P. Sas and B. Bergen (Eds.), Proceedings of the International conference on Noise and Vibration Engineering (ISMA 2010), Leuven, Belgium, 20-22 September 2010, pp. 1181-1192. 